\title{
sciendo
}

\author{
BULGARIAN ACADEMY OF SCIENCES
}

CYBERNETICS AND INFORMATION TECHNOLOGIES • Volume 20, No 6

Special Issue on New Developments in Scalable Computing

Sofia $\bullet 2020$

Print ISSN: 1311-9702; Online ISSN: 1314-4081

DOI: $10.2478 /$ cait-2020-0064

\section{Performance Analysis of a Scalable Algorithm for 3D Linear Transforms on Supercomputer with Intel Processors/Co- Processors}

\section{Ivan Lirkov}

Institute of Information and Communication, Technologies, Bulgarian Academy of Sciences, 1113 Sofia, Bulgaria

E-mail: ivan@parallel.bas.bg

\begin{abstract}
Practical realizations of $3 D$ forward/inverse separable discrete transforms, such as Fourier transform, cosine/sine transform, etc. are frequently the principal limiters that prevent many practical applications from scaling to a large number of processors. Existing approaches, which are based primarily on $1 D$ or $2 D$ data decompositions, prevent the $3 D$ transforms from effectively scaling to the maximum (possible/available) number of computer nodes. A highly scalable approach to realize forward/inverse $3 D$ transforms has been proposed. It is based on a $3 D$ decomposition of data and geared towards a torus network of computer nodes. The proposed algorithms requires compute-and-roll time-steps, where each step consists of an execution of multiple GEMM operations and concurrent movement of cubical data blocks between nearest neighbors. The aim of this paper is to present an experimental performance study of an implementation on high performance computer architecture.
\end{abstract}

Keywords: 3D linear transforms, parallel implementation, Intel processors/ co-processors.

\section{Introduction}

Three-Dimensional (3D) Discrete Transforms (DT) such as Fourier transform, cosine/sine transform, etc., are known to play a fundamental role in many application areas, such as spectral analysis, digital filtering, signal and image processing, data compression, medical diagnostics, etc. Continuously increasing demands for high speed computing in a constantly increasing number of many real-world applications have stimulated the development of a number of "fast algorithms", such as the Fast Fourier Transform (FFT), characterized by dramatic reduction of arithmetic complexity. However, further reduction of execution time is possible only by using parallel implementation. 
There exist three different approaches to parallel implementation of the 3D forward/inverse discrete transforms. Two of them are particularly well suited for the Fourier transform. The first one is the 1D or "slab" decomposition of the initial 3D data. In this approach, the data is divided into 2D slabs. The scalability of the slabbased approach is limited by the number of data elements along a single dimension of the 3D transform. The second approach is the 2D or "pencil" decomposition, of a 3D initial data, among a 2D array of computer nodes. This approach increases the maximum number of nodes that can be effectively used in computations.

The last approach is the 3D or "cube" decomposition, which was recently proposed in [1]. The 3D or "cubic" decomposition of an initial data among computer nodes, allows a 3D data "cube" to be assigned to each computer node. It is easy to realize that the theoretical scalability is further improved. In this approach, blocked GEMM-based algorithms are used to compute the basic one-dimensional $N$-size transform, not on a single but on the cyclically interconnected nodes of a 3D torus network. In this way, the proposed algorithm integrates local intra-node computation with a nearest-neighbour inter-node communication, at each step of the threedimensional processing. It is important to observe that the proposed algorithm, with its 3D data decomposition, and the torus-oriented communication scheme, completely eliminates global communication. In addition, computation and local communication can be overlapped. Finally, note that in the considered approach, the 3D transform is represented as three chained sets of cubical tensor-by-matrix or matrix-by-tensor multiplications, which are executed in a 3D torus network of computer nodes by the fastest and extremely scalable orbital algorithms.

The main contribution of this paper is to experimentally evaluate the performance of the latter algorithm. To do this, we have implemented overlapping of computation and communication for the 3D data decomposition and used GEMM kernels available. The experimental performance of the 3D Discrete Cosine Transform (DCT) and Discrete Fourier Transform (DFT), with the 3D data decomposition, has been evaluated on a supercomputer cluster.

\section{3D separable transform}

Let $X=\left[x\left(n_{1}, n_{2}, n_{3}\right)\right], 0 \leq n_{1}, n_{2}, n_{3}<N$, be an $N \times N \times N$ cubical grid of input data. A separable forward 3D transform of $X$ is another cubical grid of an $N \times N \times N$ data $Y=\left[y\left(k_{1}, k_{2}, k_{3}\right)\right]$, where for all $0 \leq k_{1}, k_{2}, k_{3}<N$ :

$$
y\left(k_{1}, k_{2}, k_{3}\right)=\sum_{n_{1}=0}^{N-1} \sum_{n_{2}=0}^{N-1} \sum_{n_{3}=0}^{N-1} x\left(n_{1}, n_{2}, n_{3}\right) \times c\left(n_{1}, k_{1}\right) \times c\left(n_{2}, k_{2}\right) \times c\left(n_{3}, k_{3}\right) .
$$

A separable inverse, or backward, 3D transform of a tensor $Y=\left[y\left(k_{1}, k_{2}, k_{3}\right)\right]$ is expressed as:

$$
x\left(n_{1}, n_{2}, n_{3}\right)=\sum_{k_{1}=0}^{N-1} \sum_{k_{2}=0}^{N-1} \sum_{k_{3}=0}^{N-1} y\left(k_{1}, k_{2}, k_{3}\right) \times c\left(n_{1}, k_{1}\right) \times c\left(n_{2}, k_{2}\right) \times c\left(n_{3}, k_{3}\right) .
$$

The 3D transform can be split into three data dependent sets of 1D transforms. At the first stage, the 1D transform of $x\left(n_{1}, n_{2},:\right)$ is performed for all $\left(n_{1}, n_{2}\right)$ pairs, as a block tensor-by-matrix multiplication. At the second stage, the 1D transform of 
$v\left(:, n_{2}, k_{3}\right)$ is implemented for all $\left(n_{2}, k_{3}\right)$ pairs, as the second block tensor-by-matrix multiplication. At the third stage, the 1D transform of $w\left(k_{1},:, k_{3}\right)$ is implemented for all $\left(k_{1}, k_{3}\right)$ pairs, as the third block tensor-by-matrix multiplication.

\section{Parallel implementation of the algorithm}

The parallel implementation of the proposed algorithm is described in [1]. Our implementation of the parallel algorithm is described in [2]. It should be noted that our implementation is a modification of the parallel algorithms proposed in [1]. The main differences between our implementation and the original algorithm are:

1. The implemented parallel algorithm works only for the 3D DCT and the 3D DFT;

2. The proposed implementation uses additional arrays to store elements of the coefficient matrix C. In the case of the DCT, we use one array with $4 N$ elements; while for the DFT two arrays with $N$ elements each. In this way, we avoid rolling the coefficient matrix. In other words, we simplify the communication, while paying the price of somewhat increasing the total memory utilization.

Since the tensor-by-matrix, or the matrix-by-tensor, multiplications can be expressed as the set of matrix-by-matrix multiplications, we can use existing GEMM subroutines, from the BLAS library [3], to compute the 3D transform.

There exist two possible ways to compute the tensor-by-matrix multiplication on computers with multi-core processors. The first one is to use a multi-threaded library, such as the Intel Math Kernel Library (MKL, see [4]). Here, each slice of the tensor is computed by multiple threads. The other possible approach is to use OpenMP. In the current implementation, we have linked our code to the multithreaded library for the parallelization on a single (multi-core) node of the computer system.

\section{Experimental results}

A portable parallel code was designed and implemented in C. The parallelization was based on the MPI standard [5,6]. In the code, we used the BLAS subroutines SGEMM, DGEMM, CGEMM, and ZGEMM to perform matrix-by-matrix multiplication. In order to obtain a better mapping of the processors to the physical interconnect topology of computers actually used in experiments, functions MPI_Dims_create and MPI_Cart_create were used to create a logical 3D Cartesian grid of processors.

The parallel code has been tested on the following system: the supercomputer Avitohol at IICT-BAS (see [7] for details). The computer system Avitohol is constructed with HP Cluster Platform SL250S GEN8. It has 150 servers, and two 8-core Intel Xeon E5-2650 v2 8C processors and two Intel Xeon Phi 7120P coprocessors per node. Each processor runs at $2.6 \mathrm{GHz}$. Processors within each node share $64 \mathrm{~GB}$ of memory. Each Intel Xeon Phi has 61 cores, runs at $1.238 \mathrm{GHz}$, and has 16 GB of memory. Nodes are interconnected with a high-speed InfiniBand FDR network. We used the Intel $\mathrm{C}$ compiler, and compiled the code using the following 
options: "-O3 -qopenmp" for the processors and "-O3 -qopenmp -mmic" for the coprocessors. To use the BLAS subroutines, we linked our code to the optimized multi-threaded Intel MKL library. Intel MPI was used to execute the code on the Avitohol computer system.

In our experiments, times have been collected using the MPI provided timer, and we report the best results from multiple runs. In the following tables, we report the elapsed (wall-clock) time in seconds.

Table 1. Execution time (in seconds) for the 3D discrete cosine transform using only processors of the Avitohol

\begin{tabular}{|c|c|c|c|c|c|c|c|}
\hline \multirow{2}{*}{$N$} & \multicolumn{7}{|c|}{ Number of nodes } \\
\hline & 1 & 2 & 4 & 8 & 16 & 32 & 64 \\
\hline \multicolumn{8}{|c|}{ Single precision } \\
\hline \multicolumn{8}{|c|}{ Forward transform } \\
\hline 200 & 0.0760 & 0.0539 & 0.0377 & 0.0383 & 0.0229 & 0.0382 & 0.0430 \\
\hline 400 & 0.7918 & 0.4543 & 0.2868 & 0.1728 & 0.1300 & 0.0953 & 0.0858 \\
\hline 600 & 3.5642 & 2.0189 & 1.1939 & 0.6426 & 0.4223 & 0.2949 & 0.2033 \\
\hline 800 & 10.5593 & 5.7550 & 3.1507 & 1.7097 & 1.0306 & 0.6472 & 0.4305 \\
\hline 1000 & 24.4799 & 13.2777 & 7.2777 & 3.9019 & 2.3329 & 1.4249 & 0.8147 \\
\hline 1200 & 49.9908 & 26.1017 & 14.1655 & 7.5324 & 4.4960 & 2.6249 & 1.4916 \\
\hline 1600 & & 78.8522 & 41.6059 & 21.7961 & 12.3199 & 6.8936 & 3.7470 \\
\hline 2000 & & & 99.5842 & 50.8931 & 28.2158 & 15.5731 & 8.6936 \\
\hline \multicolumn{8}{|c|}{ Backward transform } \\
\hline 200 & 0.0578 & 0.0365 & 0.0216 & 0.0132 & 0.0093 & 0.0109 & 0.0068 \\
\hline 400 & 0.7034 & 0.4019 & 0.2266 & 0.1300 & 0.0880 & 0.0568 & 0.0473 \\
\hline 600 & 3.3402 & 1.8719 & 1.0193 & 0.5534 & 0.3539 & 0.2301 & 0.1477 \\
\hline 800 & 9.9892 & 5.3489 & 2.8409 & 1.5194 & 0.8993 & 0.5489 & 0.3376 \\
\hline 1000 & 23.2535 & 12.5482 & 6.6254 & 3.5717 & 2.1258 & 1.2804 & 0.7387 \\
\hline 1200 & 47.9282 & 24.7840 & 13.0700 & 6.9643 & 4.1524 & 2.3548 & 1.3040 \\
\hline 1600 & & 75.7462 & 38.6973 & 20.3578 & 11.5351 & 6.5202 & 3.6148 \\
\hline 2000 & & & 94.4404 & 47.9034 & 26.8596 & 14.7392 & 8.1383 \\
\hline \multicolumn{8}{|c|}{ Double precision } \\
\hline \multicolumn{8}{|c|}{ Forward transform } \\
\hline 200 & 0.1449 & 0.0930 & 0.0646 & 0.0482 & 0.0396 & 0.0451 & 0.0540 \\
\hline 400 & 1.6184 & 0.9230 & 0.5462 & 0.3293 & 0.2197 & 0.1373 & 0.1230 \\
\hline 600 & 7.2401 & 3.9339 & 2.2233 & 1.2521 & 0.7916 & 0.4978 & 0.3228 \\
\hline 800 & 21.3262 & 11.4433 & 6.3369 & 3.4622 & 2.1033 & 1.2351 & 0.7732 \\
\hline 1000 & 51.5733 & 26.4074 & 14.4434 & 7.7706 & 4.6434 & 2.6952 & 1.6234 \\
\hline 1200 & 110.0560 & 52.9195 & 28.4283 & 15.1895 & 8.6458 & 4.9744 & 2.7664 \\
\hline 1600 & & & 87.1884 & 44.3340 & 24.5492 & 13.5643 & 7.7001 \\
\hline 2000 & & & & & 56.4890 & 30.6572 & 16.9175 \\
\hline \multicolumn{8}{|c|}{ Backward transform } \\
\hline 200 & 0.1190 & 0.0698 & 0.0393 & 0.0218 & 0.0156 & 0.0119 & 0.0104 \\
\hline 400 & 1.4522 & 0.8111 & 0.4456 & 0.2619 & 0.1689 & 0.1058 & 0.0713 \\
\hline 600 & 6.7475 & 3.6326 & 1.9578 & 1.0926 & 0.6862 & 0.4143 & 0.2865 \\
\hline 800 & 20.2844 & 10.7186 & 5.6413 & 3.0990 & 1.8295 & 1.0747 & 0.6505 \\
\hline 1000 & 49.1480 & 25.0125 & 13.0928 & 7.0599 & 4.1540 & 2.4528 & 1.4205 \\
\hline 1200 & 101.8070 & 50.5865 & 26.1044 & 13.9867 & 7.9348 & 4.5263 & 2.5333 \\
\hline 1600 & & & 82.1612 & 41.9168 & 23.0593 & 12.7839 & 7.2690 \\
\hline 2000 & & & & & 53.7557 & 29.2964 & 16.0420 \\
\hline
\end{tabular}


Tables 1 and 2 show the results collected on the Avitohol using only Intel Xeon processors. The main memory on one node is $64 \mathrm{~GB}$ and allows execution of the DCT algorithm for $N=200,400, \ldots, 1200$. The DFT algorithm requires more memory and the limit for the execution on one node is $N=1000$. For larger problems we used the distributed memory on more nodes. For example, for DFT with $N=2000$ the algorithm requires the memory of at least 16 nodes. The reported execution time for $N=200$ shows that the problem is small and can be executed on one node (no need for parallelization). Here, there is no significant improvement from using two or more nodes. However, for the problems of size $N=800,1000,1200$ a significant performance gain can be observed. Moreover, for 3D DFT with double precision super-linear speed-up is observed for $N=1000$ on up to 8 nodes.

Table 2. Execution time (in seconds) for the 3D discrete Fourier transform using only processors of the Avitohol

\begin{tabular}{|c|c|c|c|c|c|c|c|}
\hline \multirow{2}{*}{$N$} & \multicolumn{7}{|c|}{ Number of nodes } \\
\hline & 1 & 2 & 4 & 8 & 16 & 32 & 64 \\
\hline \multicolumn{8}{|c|}{ Single precision } \\
\hline \multicolumn{8}{|c|}{ Forward transform } \\
\hline 200 & 0.2067 & 0.1258 & 0.0897 & 0.0646 & 0.0493 & 0.0588 & 0.0886 \\
\hline 400 & 2.6787 & 1.4327 & 0.8654 & 0.4731 & 0.3066 & 0.1958 & 0.1228 \\
\hline 600 & 12.8205 & 6.6810 & 3.6997 & 1.8912 & 1.1365 & 0.6946 & 0.4571 \\
\hline 800 & 37.6882 & 19.9276 & 10.5935 & 5.5964 & 3.1616 & 1.8203 & 1.1089 \\
\hline 1000 & 89.3692 & 46.4069 & 24.6894 & 12.9602 & 7.2873 & 4.1502 & 2.3694 \\
\hline 1200 & & 96.4621 & 50.2716 & 26.4370 & 14.3301 & 8.0371 & 4.2275 \\
\hline 1600 & & & 152.9335 & 77.4458 & 41.4072 & 22.2047 & 12.1978 \\
\hline 2000 & & & & & 96.3475 & 51.1302 & 27.6908 \\
\hline \multicolumn{8}{|c|}{ Backward transform } \\
\hline 200 & 0.1776 & 0.1025 & 0.0579 & 0.0325 & 0.0214 & 0.0215 & 0.0158 \\
\hline 400 & 2.5317 & 1.3308 & 0.7188 & 0.3885 & 0.2352 & 0.1483 & 0.1085 \\
\hline 600 & 12.4051 & 6.5596 & 3.4169 & 1.7331 & 1.0117 & 0.5914 & 0.3761 \\
\hline 800 & 36.8481 & 19.4619 & 10.0323 & 5.2546 & 2.8643 & 1.6462 & 0.8828 \\
\hline 1000 & 87.4686 & 45.3237 & 23.2665 & 12.3150 & 6.8326 & 3.7944 & 2.2520 \\
\hline 1200 & & 93.3873 & 48.3290 & 25.3269 & 13.8271 & 7.5106 & 4.1530 \\
\hline 1600 & & & 148.0450 & 75.8290 & 40.7702 & 21.6635 & 11.8671 \\
\hline 2000 & & & & & 94.6919 & 49.9192 & 26.6086 \\
\hline \multicolumn{8}{|c|}{ Double precision } \\
\hline \multicolumn{8}{|c|}{ Forward transform } \\
\hline 200 & 0.4485 & 0.2545 & 0.1686 & 0.0971 & 0.0897 & 0.0655 & 0.0672 \\
\hline 400 & 5.9599 & 3.1502 & 1.7757 & 0.9420 & 0.5871 & 0.3953 & 0.2616 \\
\hline 600 & 28.5156 & 14.7995 & 7.7479 & 3.9093 & 2.2731 & 1.3496 & 0.7991 \\
\hline 800 & 86.9170 & 45.2880 & 23.6875 & 12.2694 & 6.7581 & 3.8764 & 2.1842 \\
\hline 1000 & 238.4780 & 107.3896 & 56.2608 & 29.2851 & 15.6836 & 8.7244 & 4.8547 \\
\hline 1200 & & & 115.2575 & 58.9024 & 30.9047 & 16.3460 & 8.6596 \\
\hline 1600 & & & & & 94.2719 & 48.8668 & 26.2570 \\
\hline \multicolumn{8}{|c|}{ Backward transform } \\
\hline 200 & 0.3976 & 0.2212 & 0.1356 & 0.0649 & 0.0455 & 0.0288 & 0.0243 \\
\hline 400 & 5.6193 & 2.9700 & 1.5695 & 0.8475 & 0.4922 & 0.2879 & 0.1865 \\
\hline 600 & 27.3639 & 14.1340 & 7.2135 & 3.6298 & 2.1130 & 1.2073 & 0.6771 \\
\hline 800 & 84.5136 & 43.6104 & 22.6527 & 11.4513 & 6.2700 & 3.5147 & 2.1582 \\
\hline 1000 & 216.1370 & 104.4850 & 53.0580 & 27.9994 & 14.9497 & 8.3255 & 4.6314 \\
\hline 1200 & & & 110.3050 & 56.8326 & 29.5519 & 15.6329 & 8.2157 \\
\hline 1600 & & & & & 91.7581 & 47.6514 & 25.0236 \\
\hline
\end{tabular}


Tables 3 and 4 contain the execution time using only Intel Xeon Phi. Here one can observe that the algorithms run faster using one coprocessor than using 2, 4, 8 coprocessors. It is clear that the communication time between coprocessors is large and there is no improvement using the parallel implementation of the algorithms.

Table 3. Execution time (in seconds) for the 3D discrete cosine transform using only coprocessors of the Avitohol

\begin{tabular}{|c|c|c|c|c|c|c|c|}
\hline \multirow{2}{*}{$N$} & \multicolumn{7}{|c|}{ Number of coprocessors } \\
\hline & 1 & 2 & 4 & 8 & 16 & 32 & 64 \\
\hline \multicolumn{8}{|c|}{ Single precision } \\
\hline \multicolumn{8}{|c|}{ Forward transform } \\
\hline 200 & 0.5124 & 0.6388 & 0.6370 & 0.4128 & 0.3938 & 0.3576 & 0.2495 \\
\hline 400 & 1.2582 & 1.9077 & 1.9876 & 1.4857 & 1.3592 & 1.1430 & 0.7298 \\
\hline 600 & 3.7672 & 5.4232 & 5.4707 & 3.8514 & 3.5044 & 2.7911 & 1.8514 \\
\hline 800 & 8.5344 & 13.5098 & 11.9203 & 8.0540 & 7.5502 & 5.7663 & 3.8314 \\
\hline 1000 & & 26.7401 & 23.8716 & 16.2240 & 14.3935 & 10.6471 & 6.9524 \\
\hline 1200 & & & 41.1496 & 29.6708 & 24.2277 & 18.2094 & 11.7408 \\
\hline 1600 & & & & 69.3336 & 59.1269 & 41.4999 & 26.6460 \\
\hline 2000 & & & & & 115.3765 & 81.7667 & 52.4187 \\
\hline \multicolumn{8}{|c|}{ Backward transform } \\
\hline 200 & 0.0642 & 0.1746 & 0.2181 & 0.1598 & 0.1425 & 0.1228 & 0.0836 \\
\hline 400 & 0.4447 & 1.3052 & 1.4283 & 1.0150 & 0.9230 & 0.7501 & 0.4711 \\
\hline 600 & 1.8362 & 4.4320 & 4.5303 & 3.3942 & 3.0188 & 2.3530 & 1.4862 \\
\hline 800 & 4.8155 & 11.4296 & 10.4895 & 7.7898 & 6.9923 & 5.3358 & 3.3693 \\
\hline 1000 & & 23.5476 & 21.2399 & 15.2939 & 13.6295 & 10.2196 & 6.4541 \\
\hline 1200 & & & 36.8365 & 27.3000 & 23.6922 & 17.5493 & 11.2333 \\
\hline 1600 & & & & 64.4491 & 57.4970 & 40.9009 & 26.0027 \\
\hline 2000 & & & & & 112.4650 & 80.4906 & 51.5106 \\
\hline \multicolumn{8}{|c|}{ Double precision } \\
\hline \multicolumn{8}{|c|}{ Forward transform } \\
\hline 200 & 0.5582 & 0.7781 & 0.8064 & 0.5260 & 0.5054 & 0.4507 & 0.3163 \\
\hline 400 & 2.5085 & 3.3958 & 3.2535 & 2.4080 & 2.2024 & 1.7706 & 1.1271 \\
\hline 600 & 7.7727 & 11.1460 & 10.3057 & 7.0143 & 6.3357 & 4.7157 & 3.1787 \\
\hline 800 & & 27.1808 & 24.2012 & 16.1103 & 14.4354 & 10.5462 & 6.9842 \\
\hline 1000 & & & 48.4785 & 34.2117 & 28.2551 & 20.3837 & 13.1819 \\
\hline 1200 & & & & 59.5258 & 49.7826 & 35.1730 & 22.3118 \\
\hline 1600 & & & & & 118.5665 & 83.4042 & 53.2028 \\
\hline 2000 & & & & & & & 105.3077 \\
\hline \multicolumn{8}{|c|}{ Backward transform } \\
\hline 200 & 0.0929 & 0.3182 & 0.3482 & 0.2501 & 0.2369 & 0.2030 & 0.1229 \\
\hline 400 & 0.9738 & 2.5964 & 2.6336 & 1.9374 & 1.7742 & 1.3468 & 0.8450 \\
\hline 600 & 4.4812 & 9.3757 & 8.9153 & 6.5473 & 5.8316 & 4.3498 & 2.7440 \\
\hline 800 & & 24.3902 & 21.5345 & 15.2626 & 13.9939 & 10.1905 & 6.5200 \\
\hline 1000 & & & 42.7577 & 32.2109 & 27.9494 & 19.8042 & 12.6917 \\
\hline 1200 & & & & 56.4205 & 48.3041 & 34.6965 & 21.8462 \\
\hline 1600 & & & & & 117.8540 & 82.2662 & 52.1192 \\
\hline 2000 & & & & & & & 104.8230 \\
\hline
\end{tabular}


Table 4. Execution time (in seconds) for the 3D discrete Fourier transform using only coprocessors of the Avitohol

\begin{tabular}{|c|c|c|c|c|c|c|c|}
\hline \multirow{2}{*}{$N$} & \multicolumn{7}{|c|}{ Number of coprocessors } \\
\hline & 1 & 2 & 4 & 8 & 16 & 32 & 64 \\
\hline \multicolumn{8}{|c|}{ Single precision } \\
\hline \multicolumn{8}{|c|}{ Forward transform } \\
\hline 200 & 0.6733 & 0.8424 & 0.8686 & 0.5990 & 0.5611 & 0.5129 & 0.3537 \\
\hline 400 & 2.6020 & 3.6413 & 3.5441 & 2.4803 & 2.2891 & 1.8462 & 1.1848 \\
\hline 600 & 8.1777 & 11.9750 & 10.3334 & 7.2245 & 6.5103 & 4.9759 & 3.2558 \\
\hline 800 & & 27.8812 & 24.3975 & 16.9994 & 14.7433 & 10.8582 & 7.0387 \\
\hline 1000 & & & 49.5779 & 35.6633 & 28.9276 & 20.9952 & 13.5672 \\
\hline 1200 & & & & 61.3304 & 49.5451 & 35.3441 & 22.8684 \\
\hline 1600 & & & & & 118.7209 & 83.9047 & 53.5311 \\
\hline 2000 & & & & & & & 105.9995 \\
\hline \multicolumn{8}{|c|}{ Backward transform } \\
\hline 200 & 0.1423 & 0.3791 & 0.4080 & 0.3115 & 0.2805 & 0.2506 & 0.1830 \\
\hline 400 & 1.1092 & 2.7578 & 2.7353 & 1.9834 & 1.8473 & 1.4136 & 0.9093 \\
\hline 600 & 4.7513 & 9.6823 & 9.1412 & 6.6159 & 6.0284 & 4.4975 & 2.8664 \\
\hline 800 & & 24.6317 & 21.8166 & 16.1799 & 14.0978 & 10.3299 & 6.5010 \\
\hline 1000 & & & 44.4229 & 32.3628 & 27.8660 & 19.8807 & 12.8469 \\
\hline 1200 & & & & 57.0783 & 48.8767 & 34.6318 & 22.2119 \\
\hline 1600 & & & & & 116.7550 & 82.6160 & 52.6932 \\
\hline 2000 & & & & & & & 104.9290 \\
\hline \multicolumn{8}{|c|}{ Double precision } \\
\hline \multicolumn{8}{|c|}{ Forward transform } \\
\hline 200 & 0.8991 & 1.1888 & 1.2154 & 0.7955 & 0.7516 & 0.6587 & 0.4543 \\
\hline 400 & 4.9907 & 7.0999 & 6.4597 & 4.4377 & 3.9776 & 3.0814 & 1.9723 \\
\hline 600 & & 24.1780 & 21.1313 & 14.2214 & 12.7873 & 9.0109 & 5.9362 \\
\hline 800 & & & 51.2982 & 36.0511 & 29.5805 & 21.0108 & 13.5255 \\
\hline 1000 & & & & 74.3123 & 58.3255 & 41.1495 & 26.7309 \\
\hline 1200 & & & & & 102.2205 & 71.1527 & 45.5205 \\
\hline \multicolumn{8}{|c|}{ Backward transform } \\
\hline 200 & 0.3142 & 0.7203 & 0.7460 & 0.5714 & 0.5261 & 0.4479 & 0.2994 \\
\hline 400 & 2.6119 & 5.6940 & 5.4064 & 3.9828 & 3.5879 & 2.6907 & 1.7472 \\
\hline 600 & & 21.6765 & 18.6295 & 13.3133 & 11.8331 & 8.7192 & 5.6127 \\
\hline 800 & & & 45.0476 & 32.8309 & 28.7036 & 20.6122 & 13.1516 \\
\hline 1000 & & & & 67.0370 & 56.7831 & 40.8836 & 25.7711 \\
\hline 1200 & & & & & 100.3330 & 70.5225 & 44.7571 \\
\hline
\end{tabular}


Tables 5 and 6 present times collected on the Avitohol using processors as well as coprocessors. We made the experiments using 4 MPI processes on each node: 2 processes on processors and 2 processes on coprocessors. The results in Table 5 show that again there is no improvement using the parallel implementation of the algorithm. Conversely, we can see from Table 6 the execution time of the parallel implementation of the algorithm for 3D DFT is better than the time of the sequential algorithm.

Table 5. Execution time (in seconds) for the 3D discrete cosine transform using processors and coprocessors of the Avitohol

\begin{tabular}{|c|c|c|c|c|c|c|}
\hline \multirow{2}{*}{$N$} & \multicolumn{6}{|c|}{ Number of nodes } \\
\hline & 1 & 2 & 4 & 8 & 16 & 32 \\
\hline \multicolumn{7}{|c|}{ Single precision } \\
\hline \multicolumn{7}{|c|}{ Forward transform } \\
\hline 200 & 1.0461 & 0.9174 & 0.7746 & 0.6689 & 0.5356 & 0.5219 \\
\hline 400 & 5.7840 & 3.7579 & 2.5590 & 1.8448 & 1.1934 & 0.9889 \\
\hline 600 & 19.8967 & 11.1379 & 7.0881 & 4.5794 & 2.9457 & 2.0704 \\
\hline 800 & 47.7289 & 27.0011 & 15.7896 & 9.7563 & 5.9931 & 3.9820 \\
\hline 1000 & 96.2926 & 54.0093 & 31.1242 & 18.5277 & 10.9908 & 7.0737 \\
\hline 1200 & 172.6714 & 94.5210 & 54.0907 & 31.5976 & 18.3560 & 11.6593 \\
\hline 1600 & & 244.7497 & 134.0510 & 76.1986 & 42.2924 & 26.2381 \\
\hline 2000 & & & 272.4915 & 152.2338 & 83.7523 & 51.7131 \\
\hline \multicolumn{7}{|c|}{ Backward transform } \\
\hline 200 & 0.3160 & 0.0430 & 0.0309 & 0.0620 & 0.0742 & 0.0825 \\
\hline 400 & 1.6914 & 0.6862 & 0.6055 & 0.4975 & 0.3279 & 0.2435 \\
\hline 600 & 4.2980 & 2.6665 & 2.3685 & 1.8131 & 1.1951 & 0.8605 \\
\hline 800 & 9.2476 & 6.1460 & 6.0348 & 4.4966 & 2.7682 & 2.0479 \\
\hline 1000 & 19.5277 & 13.0295 & 12.2256 & 9.0832 & 5.3651 & 3.9084 \\
\hline 1200 & 33.4088 & 22.9409 & 20.9885 & 16.0037 & 9.2687 & 6.9492 \\
\hline 1600 & & 57.2751 & 51.6913 & 37.6339 & 21.8078 & 16.4330 \\
\hline 2000 & & & 101.5010 & 75.0368 & 43.7860 & 33.0999 \\
\hline \multicolumn{7}{|c|}{ Double precision } \\
\hline \multicolumn{7}{|c|}{ Forward transform } \\
\hline 200 & 1.1424 & 0.9993 & 0.8713 & 0.7596 & 0.6030 & 0.5582 \\
\hline 400 & 6.4779 & 4.4091 & 3.2350 & 2.3677 & 1.5319 & 1.2655 \\
\hline 600 & 23.4372 & 14.2287 & 9.4932 & 6.2915 & 4.0761 & 2.9181 \\
\hline 800 & 57.5915 & 34.4666 & 21.7705 & 13.9947 & 8.6594 & 5.9716 \\
\hline 1000 & 119.0888 & 69.6325 & 43.1526 & 27.0916 & 16.0252 & 10.8204 \\
\hline 1200 & & 120.6609 & 76.2304 & 47.3631 & 27.6925 & 18.0780 \\
\hline 1600 & & & 193.9048 & 114.2277 & 65.3929 & 42.6685 \\
\hline 2000 & & & & & 130.9305 & 86.1398 \\
\hline \multicolumn{7}{|c|}{ Backward transform } \\
\hline 200 & 0.5059 & 0.0926 & 0.0493 & 0.0863 & 0.0590 & 0.0650 \\
\hline 400 & 2.8307 & 1.4440 & 1.3961 & 0.9820 & 0.6129 & 0.4586 \\
\hline 600 & 8.1704 & 5.2758 & 5.0683 & 3.7362 & 2.2408 & 1.6889 \\
\hline 800 & 19.7701 & 13.2111 & 12.4784 & 9.2636 & 5.4426 & 3.9528 \\
\hline 1000 & 39.2059 & 26.1137 & 24.7823 & 18.1029 & 10.6478 & 7.8551 \\
\hline 1200 & & 45.5004 & 43.6663 & 31.7628 & 18.3667 & 13.7592 \\
\hline 1600 & & & 104.6020 & 75.7410 & 43.5752 & 32.6848 \\
\hline 2000 & & & & & 87.7772 & 63.9718 \\
\hline
\end{tabular}


Table 6. Execution time (in seconds) for the 3D discrete Fourier transform using processors and coprocessors of the Avitohol

\begin{tabular}{|c|c|c|c|c|c|c|}
\hline \multirow{2}{*}{$N$} & \multicolumn{6}{|c|}{ Number of nodes } \\
\hline & 1 & 2 & 4 & 8 & 16 & 32 \\
\hline \multicolumn{7}{|c|}{ Single precision } \\
\hline \multicolumn{7}{|c|}{ Forward transform } \\
\hline 200 & 0.7257 & 0.8685 & 0.8249 & 0.7840 & 0.6487 & 0.6235 \\
\hline 400 & 3.3775 & 2.8575 & 2.3905 & 1.9840 & 1.4639 & 1.2543 \\
\hline 600 & 11.8316 & 8.5432 & 6.5842 & 4.8935 & 3.3939 & 2.6014 \\
\hline 800 & 31.0851 & 21.1444 & 15.2111 & 10.7736 & 6.8629 & 5.1113 \\
\hline 1000 & 67.0818 & 43.5047 & 30.1038 & 20.7041 & 12.9051 & 9.3361 \\
\hline 1200 & & 79.1054 & 54.5836 & 35.8087 & 22.2026 & 15.6287 \\
\hline 1600 & & & 144.7684 & 87.8005 & 53.2528 & 36.7738 \\
\hline 2000 & & & & & 107.5775 & 74.2922 \\
\hline \multicolumn{7}{|c|}{ Backward transform } \\
\hline 200 & 0.6455 & 0.2580 & 0.0944 & 0.2067 & 0.1816 & 0.1652 \\
\hline 400 & 2.7533 & 1.5852 & 1.5336 & 1.1868 & 0.7561 & 0.5598 \\
\hline 600 & 8.2752 & 5.4929 & 5.3142 & 3.9293 & 2.3069 & 1.7960 \\
\hline 800 & 21.0940 & 14.5966 & 12.8842 & 9.4114 & 5.4024 & 4.0734 \\
\hline 1000 & 45.6085 & 29.0226 & 25.4616 & 18.8960 & 10.9276 & 7.7307 \\
\hline 1200 & & 50.1291 & 41.9014 & 32.7591 & 18.8841 & 13.7003 \\
\hline 1600 & & & 102.9000 & 76.8544 & 44.2353 & 33.2393 \\
\hline 2000 & & & & & 89.3026 & 65.2866 \\
\hline \multicolumn{7}{|c|}{ Double precision } \\
\hline \multicolumn{7}{|c|}{ Forward transform } \\
\hline 200 & 0.8904 & 1.0013 & 0.9526 & 0.8867 & 0.7206 & 0.6537 \\
\hline 400 & 5.9818 & 4.5343 & 3.8480 & 2.9957 & 2.0940 & 1.7015 \\
\hline 600 & 22.0847 & 15.3617 & 12.3924 & 8.7832 & 5.6635 & 4.2174 \\
\hline 800 & 60.0458 & 38.9667 & 29.2292 & 20.1616 & 12.8326 & 9.1372 \\
\hline 1000 & & 79.0519 & 60.2493 & 40.4552 & 25.1706 & 17.5858 \\
\hline 1200 & & & 107.1120 & 70.7953 & 43.2693 & 31.0853 \\
\hline 1600 & & & & & 104.4458 & 73.8282 \\
\hline \multicolumn{7}{|c|}{ Backward transform } \\
\hline 200 & 1.0100 & 0.3795 & 0.3436 & 0.3092 & 0.2282 & 0.2239 \\
\hline 400 & 4.9562 & 3.2677 & 2.9362 & 2.2727 & 1.4620 & 1.1017 \\
\hline 600 & 17.4575 & 11.6983 & 10.7004 & 7.9430 & 4.6395 & 3.4696 \\
\hline 800 & 45.2816 & 28.4348 & 25.3424 & 18.9955 & 10.9793 & 8.1076 \\
\hline 1000 & & 59.8817 & 50.8526 & 38.1525 & 21.9127 & 15.8757 \\
\hline 1200 & & & 87.3476 & 66.0734 & 38.0652 & 27.1347 \\
\hline 1600 & & & & & 90.2499 & 66.7974 \\
\hline
\end{tabular}

Finally, in Fig. 1, we represent execution time of the code, which performs one forward and one backward DFT. Results are presented for single and double precision, for problems of size $N=1000$ and $N=1600$. 


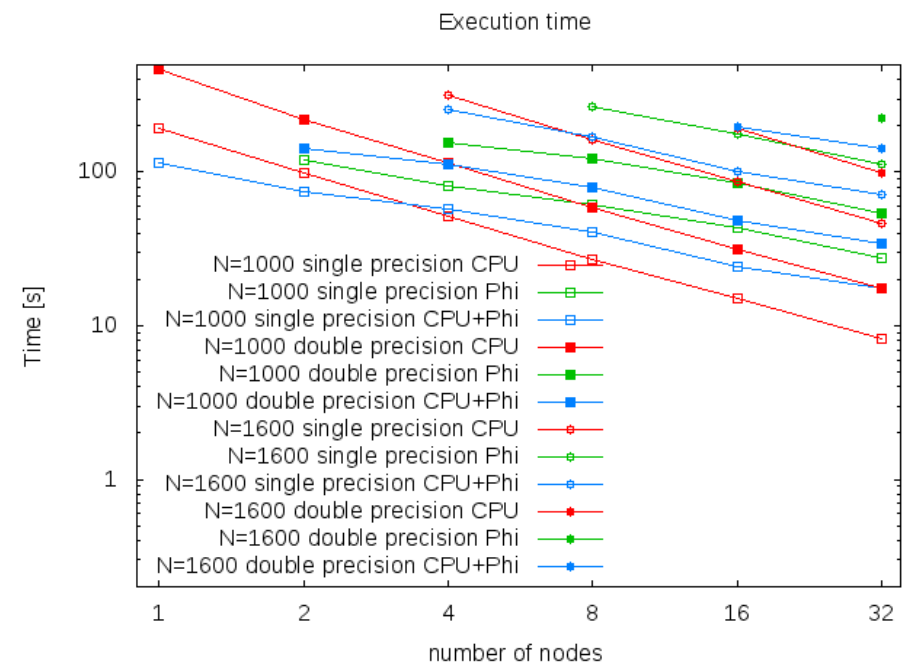

Fig. 1. Execution time of code which performs forward and backward DFT for $N=1000,1600$

\section{Conclusion}

The aim of this paper was to analyze an implementation of a slightly simplified version of an algorithm for 3D forward/inverse discrete transforms on supercomputer with Intel processors/co-processors. The algorithm was designed for a 3D torus network. The results on the supercomputer Avitohol show that a fast communication network is needed in order to obtain parallel efficiency of the algorithm. On the basis of the obtained results we can conclude that the proposed approach allows solution of large $3 \mathrm{D}$ problems on a supercomputer.

Acknowledgments: This research was partially supported by grant DNTS/Russia 02/7 from the Bulgarian NSF. This work has been accomplished with the partial support by the Grant No. BG05M2OP001-1.001-0003, financed by the Science and Education for Smart Growth Operational Program (2014-2020) and co-financed by the European Union through the European structural and Investment funds.

\section{References}

1. S e d u k h i n, S. G. Co-Design of Extremely Scalable Algorithms/Architecture for 3-Dimensional Linear Transforms. Technical Report TR2012-001. The University of Aizu, July 2012.

2. Lirkov, I., M. Paprzycki, M. Ganzha, S. Sedukhin, P. Gepner. Performance Analysis of Scalable Algorithms for 3D Linear Transforms. - In: Proc. of M. Ganzha, L. Maciaszek, M. Paprzycki, Eds. 2014 Federated Conference on Computer Science and Information Systems, Annals of Computer Science and Information Systems, Vol. 2, IEEE, 2014, pp. 613-622. DOI: 10.15439/2014F374.

3. Dongarra, J. J., J. D u C ro z, S. H a m marling, I. S. D u f f. A Set of Level 3 Basic Linear Algebra Subprograms. - ACM Transactions On Mathematical Software (TOMS), Vol. 16, 1990, No 1, pp. 1-17. DOI: 10.1145/77626.79170. 
4. Intel Math Kernel Library. http://software.intel.com/en-us/articles/intel-mkl/

5. S n i r, M., S. Ot to, S. Hu s s-Le d e r m a n, D. W a l ke r, J. D o n g a r r a. MPI: The Complete Reference. Second Edition. Vol. 1. The MPI Core. Scientific and Engineering Computation Series, Cambridge, Massachusetts, MIT Press, 1998. ISBN: 9780262692151.

6. W a $1 \mathrm{ke} \mathrm{r}$, D., J. D o n g a r r a. MPI: A Standard Message Passing Interface. - Supercomputer, Vol. 12, 1996, No 1, pp. 56-68. ISSN 0168-7875.

7. High-Performance Computing System - AVITOHOL. http://www.hpc.acad.bg/avitohol/

Received: 15.09.2020; Second Version: 19.10.2020; Accepted: 23.10.2020 